\title{
Belphégor
}

Littérature populaire et culture médiatique

15-1| 2017

1936: les Jeux olympiques dans la presse

internationale

\section{Veni, bibi, vici}

Les aventures extraordinaires de Mrs Eleanor Holm Jarrett

\section{Amélie Chabrier}

\section{(2) OpenEdition}

\section{Journals}

Electronic version

URL: http://journals.openedition.org/belphegor/913

DOI: 10.4000/belphegor.913

ISSN: 1499-7185

Publisher

LPCM

Electronic reference

Amélie Chabrier, « Veni, bibi, vici », Belphégor [Online], 15-1 | 2017, Online since 04 July 2017

connection on 19 April 2019. URL : http://journals.openedition.org/belphegor/913 ; DOI : 10.4000/

belphegor.913

This text was automatically generated on 19 April 2019

\section{$\Theta \Theta \Theta \Theta$}

Belphégor est mis à disposition selon les termes de la Licence Creative Commons Attribution - Pas d'Utilisation Commerciale - Pas de Modification 4.0 International. 


\title{
Veni, bibi, vici
}

\author{
Les aventures extraordinaires de Mrs Eleanor Holm Jarrett
}

\section{Amélie Chabrier}

Lorsque l'on parcourt la presse française sur les Jeux olympiques de Berlin, difficile de ne pas croiser un article sur l'exploit de Mrs Eleanor Holm Jarrett qui s'inscrit dans une discipline un peu particulière : la descente de champagne. En devenant la première et unique athlète de la délégation américaine expulsée pour avoir trop fréquenté le bar du Manhattan, la championne de natation devient une figure incontournable de ces jeux sans même avoir à y participer. Ainsi, dès le 25 juillet, L'Auto annonce la nouvelle, relayé le lendemain par Le Populaire, puis Le Petit Parisien, Le Figaro, L'Intransigeant... Dans cette période qui précède immédiatement l'ouverture des Jeux, l'anecdote offre une distraction de choix, avec sa teneur en drame et en cocasserie. On rivalise d'ingéniosité pour évoquer le fait, de la version euphémistique des "quelques coupes» du Figaro à l'hyperbole goguenarde "des cuites carabinées" de L'Auto. Miss Jarrett devient ainsi un petit personnage récurrent durant toute la durée des Jeux, reparaissant soit dans des brèves qui lui sont consacrées, soit dans des allusions amusées au milieu d'autres articles. Or à cette information somme toute elliptique traitée sur un mode sensationnaliste en France correspond un véritable séisme médiatique du côté américain. Miss Jarrett est partout, dans tous les journaux mais aussi dans toutes les rubriques, à l'image du numéro du 25 juillet de New York Times qui ne lui consacre pas moins de quatre articles! L'information, pléthorique et redondante outre-Atlantique, alimente les articles français qui semblent puiser à cette source intarissable pendant plusieurs semaines au quotidien. Mais une telle anecdote n'aurait jamais fait autant de vagues si son héroïne ne jouissait pas en amont d'un certain renom. Cet article souhaite donc retracer la carrière médiatique de la pétillante nageuse, en montrant comment, pour reprendre la terminologie de Nathalie Heinich, la jeune femme a cumulé différents « capitaux de visibilité ${ }^{1}$ » : en effet, Eleanor est d'abord connue grâce à ses capacités propres dans sa discipline, avant que le hasard ne la précipite, en juillet 1936, à la une de la presse internationale ; riche de cette intense médiatisation, elle en récolte les fruits encore bien après la fin des Jeux olympiques. Ainsi, si les articles français la concernant se concentrent essentiellement sur le fait divers, une 
plongée dans les archives en ligne de la presse américaine ${ }^{2}$ permet de mieux cerner l'ampleur du phénomène.

\section{Naissance de Vénus}

2 La première apparition de Miss Holm dans le New York Times date de 1927 et correspond à ses débuts en compétition, alors qu'elle n'a que douze ans. Le 21 mai, lors de sa première victoire, son nom figure en gros titre dans une page sportive : « Little Miss Holm takes 500 Yard Title ${ }^{3} »$. Durant ces années (1927-1932), le jeune âge de la nageuse est systématiquement rappelé au regard de ses prouesses sportives. L'épithète " little », qui convient aussi bien à l'âge de la nageuse qu'à son gabarit est antéposée à son nom, composant presque un surnom. La natation comme la gymnastique sont des sports dans lesquels il n'est pas rare de rencontrer de très jeunes athlètes de haut niveau et la presse recourt facilement au mythe de l'enfant prodige pour les médiatiser car tel David remportant la lutte contre Goliath, ils suscitent aisément l'adhésion, la sympathie et l'intérêt du public. Ainsi l'on retrouve souvent le substantif «marvel» pour désigner Eleanor : "the thirteen year old marvel of the Women's Swimming Association ${ }^{4}$ » ou encore lors de sa première sélection aux JO «the diminutive swimming marvel ${ }^{5}$ », la «merveilleuse petite nageuse miniature ».

3 C'est par ce biais que le premier article de Match l'introduit en France, en avril 1928, à l'occasion de son premier record du monde en triple nage : « haute à peine d'un mètre cinquante et pesant quarante et un kilos, Eleanor est bien une enfant [...]. Mais les temps qu'elle réalise laisseront rêveur plus d'un homme aux muscles respectables ${ }^{6} »$. L'article du journaliste et écrivain Henry Musnik commence par un éloge de l'exceptionnalité du phénomène. Être détenteur d'un tel record, c'est déjà un exploit, mais à cela s'ajoutent deux facteurs qui doivent forcer l'admiration: Eleanor n'a que quatorze ans et c'est une petite fille. Il commence alors à raconter le merveilleux destin, la « success story » de la "sirène enfantine »: c'est en assistant un jour à une fête de natation, qu'eut lieu la révélation. La petite Eleanor voulait nager ${ }^{7}$. Au départ, rien ne la prédestinait à un tel sport, mais grâce à son entrainement et à sa ténacité, Eleanor fit des progrès fulgurants. Dans ce portrait, le journaliste ne s'en tient pas à ses derniers résultats ou à sa préparation physique. On apprend que la jeune fille est également « remarquable en course à pied», ce qui aurait pu en faire une "spécialiste du soulier à pointe». Cette excellence dépasse donc le cadre de la natation, et même celui du sport puisque « elle est notée parmi les meilleures élèves de sa classe » et qu'elle étudie le chant et " possède une voix de rossignol»! Enfin, Henry Musnik insiste par une anecdote sur ses qualités morales de «bonne petite camarade »:

Lorsqu'elle battit Adélaïde Lambert, sa compagne de club, pulvérisant le record mondial de trois nages, elle éclata en sanglots à l'arrivée! ...Elle fut longue à se consoler d'avoir fait de la peine à celle qu'elle avait vaincue... elle affirme avec toute la candeur de ses quatorze ans que si le fait d'être championne devait lui aliéner l'affection de ses amies, elle préférait renoncer aux compétitions. ${ }^{8}$

Dans cet extrait ressort d'un côté la force de la « championne », par le recours au champ lexical du combat (battre, pulvériser, vaincre, compétitions) et de l'autre la fragilité et la douceur de la petite fille modèle (sanglots, peine, candeur, affection). Un esprit sain (et saint!) dans un corps sain pourrait-on dire, pour Eleanor, dont le conte fait ressortir des valeurs positives liées au monde du sport, comme celles du travail et de la générosité (ou 
fair-play). C'est donc sous les traits de l'enfant-prodige qu'Eleanor Holm est présentée aux lecteurs français. Or rapidement un autre facteur accroît tacitement sa visibilité outreatlantique : sa beauté9.

5 Au fil de ses exploits sportifs, la jeune nageuse grandit, suivie de près par la presse américaine. De fait, ce sont d'autres épithètes qui commencent à fleurir pour la désigner, renvoyant non plus à son jeune âge mais à son physique agréable, ce qui n'a rien d'exceptionnel là encore dans le domaine de la natation, les corps très exposés entraînant facilement ce type de commentaires. Le mythe de l'enfant-prodige cède donc la place à celui de la sirène (les termes de «mermaid » et surtout de «naiad» sont omniprésents), non pas moins stéréotypé. Les épithètes renvoyant à sa beauté sont d'autant plus nombreuses qu'en 1932, lorsqu'elle devient championne olympique pour la première fois, Eleanor signe également un contrat avec Hollywood, marchant sur les traces d'autres célèbres nageuses américaines qui ont fait carrière au cinéma, Miss Madison, Miss Roleman ou Gertrude Ederlé ${ }^{10}$.

6 Alors que Match en août 1932 ne cite son nom que dans le palmarès des JO de Los Angeles sans plus de détail, en mars 1933 le magazine sportif français par la plume du même Henry Musnik lui consacre de nouveau un récit dithyrambique dans la rubrique « le sport et la vie ", lié à ce changement de carrière. Le contraste entre la photo qui accompagne l'article de 1928 et celles de 1933 est saisissant : sur la première, Eleanor est au bord d'un bassin, mains sur les genoux, semblant reprendre son souffle après un effort. Match nous montre bien une fillette aux allures de garçonnet, dans un cliché complémentaire de l'article évoqué plus haut. Il est d'ailleurs intéressant de la comparer à la photo de Ralph Finnagan, nageur dont la pose et le costume une pièce sont quasi similaires. Au contraire, quelques années plus tard, les photos d'Eleanor mettent en valeur sa féminité. En 1934 elle pose au sortir du bassin, sourire charmeur aux lèvres dans une posture sophistiquée, malgré la tenue de bain peu seyante.

Quant aux photos publiées dans Match, ce sont celles d'une femme fatale, d'une «star »: Eleonor est montrée en tenue de soirée successivement au milieu d'autres nageuses actrices et en gros plan, fixant l'objectif d'un regard de braise, les yeux noir charbon, dans une posture qui n'a plus rien du jeune « rossignol » ou de la «bonne petite camarade»: «Eleanor Holm, nageuse et star ${ }^{11}$ ", telle est la légende qui résume bien le double capital de visibilité de la jeune femme selon les termes de Nathalie Heinich, reconnue à la fois pour ses exploits et ses atouts physiques. 


$$
\text { 䀮 }
$$




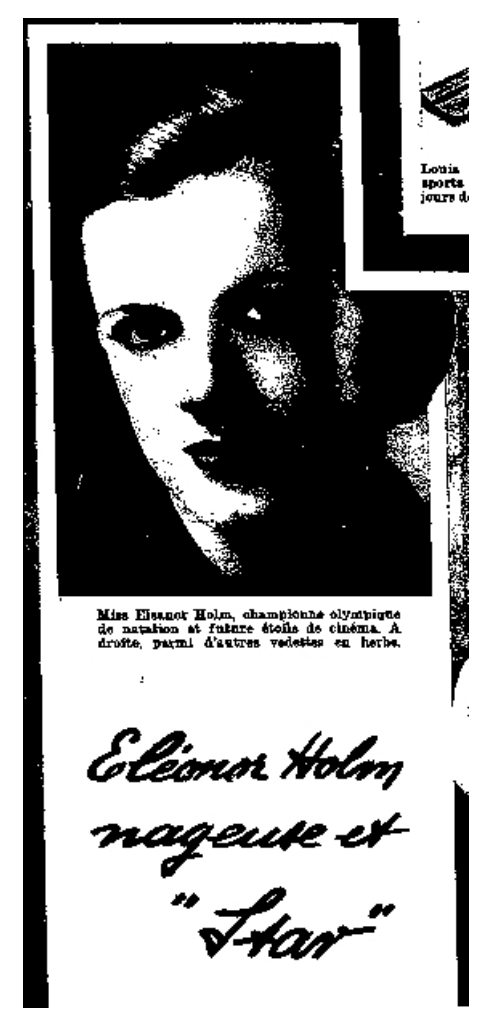

De gauche à droite, Match, 24 avril 1928 ; New York Times, 31 décembre 1934 ; Match, 7 mars 1933.

8 Néanmoins ces éléments ne sont pas rares dans le monde de la natation féminine. S'ils permettent à la jeune femme une médiatisation constante et régulière, rythmée par les compétitions sportives, ils ne la distinguent pas franchement du groupe de nageuses qui partage avec elle les mêmes qualités. Ainsi en septembre 1935 trouve-t-on dans le journal régional The Advanced News un article sur les « reines de beauté du sport ${ }^{12}$ » dans lequel elle figure aux côtés de ses coéquipières et rivales, notamment les sœurs Kompa. Cependant un dernier élément plus singulier va lui assurer à partir de 1933 une place systématique dans les gros titres: sa "peopolisation» grâce à son mariage avec le chanteur et musicien Arthur ( Art ») Jarrett.

9 Ce n'est certes pas la première fois que la page «sport " prend des allures de chronique matrimoniale. Le New York Times en août 1933 publie ainsi une brève sur son intention de se marier à Art Jarrett, et confirme un mois plus tard dans ses « social news » qu'elle est devenue sa femme. En revanche ce qui rend cette union remarquable est la célébrité des deux époux et la confrontation de deux sphères très différentes et $a$ priori inconciliables : le monde de la musique qui est celui de la nuit, de la fête, de l'excès et celui du sport, où règnent discipline, ordre, rigueur. Cette tension qui heurte la bonne morale est largement soulignée par la presse américaine, d'autant plus que la nageuse met entre parenthèses pendant un an sa carrière pour suivre son chanteur de mari et... chanter à ses côtés! Si bien qu'à son retour, Eleanor est attendue au tournant, avec cette question clé qui soustend la majorité des articles : malgré sa « mauvaise » vie si contradictoire avec celle d'une sportive de haut niveau, parviendra-t-elle à regagner la plus haute marche du podium, elle qui en sept ans n'a essuyé aucune défaite ? Et surtout à partir de mai 1936 : sera-t-elle qualifiée pour les Jeux olympiques de Berlin? Le New York Times suit de façon quasi quotidienne son retour, insistant systématiquement sur la dichotomie qui la particularise : «Her entry testifies that the champion who relinquished her titles to join 
her husband on an extensive barnstorming vaudeville and nightclub tour last year, is back in serious training ${ }^{13}$." Or Eleanor reprend rapidement la tête du championnat, reconfigurant ainsi son statut d'exceptionnalité en devenant cette fois une figure atypique, qui se démarque des autres nageuses. C'est ce que montre un article de The Advanced News qui explique qu'elle est la première à avoir réussi à la fois dans la chanson et dans le sport:

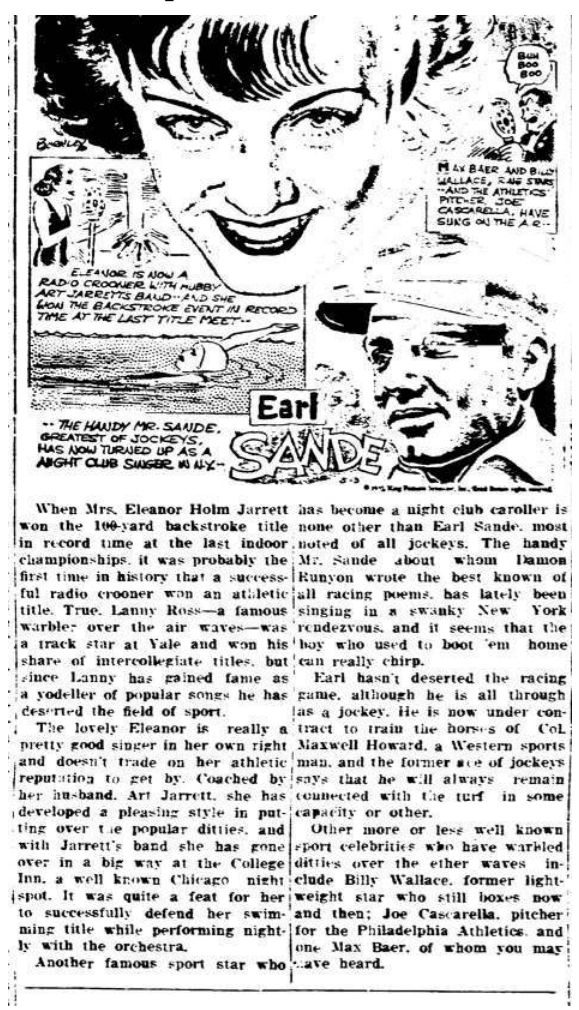

The Advanced News, 4 mai 1935. Eleanor is now a radio crooner with hubby art jarrett band... AND she won the backstroke event in record time at the last meet...

La rubrique dominicale «News of Women In Sports » du New York Times, écrite par Maribel $\mathrm{Y}$. Vinson, ancienne championne olympique de patinage artistique, montre aussi sous un jour très positif la conciliation de l'inconciliable, qui s'oppose à l'image traditionnelle de la gentille et jolie petite nageuse: «Brunette, vivacious, whose combined career of Olympic back stroke swimmer and night-club singer with her husband's orchestra, she is one of the most striking achievements in women's sports ${ }^{14}$. Ainsi c'est toujours sous le signe du «don » qu'Eleanor est présentée : « Mrs Jarrett is favored. Mrs Holm Jarrett is one of those natural athletes who can reach top of form much quicker than the ordinary competitor ${ }^{15} »$. Enfin sous la plume d'une autre femme, Eleanor semble incarner une certaine image de l'émancipation féminine.

On constate enfin qu'à partir de cette période les clichés instantanés en piscine laissent place à des poses retravaillées : Mrs Jarrett mime gracieusement la position du plongeon, mais maquillée, coiffée d'un bandeau et dans un maillot de bain de loisir qui ne nuisent en rien à son sex appeal. 


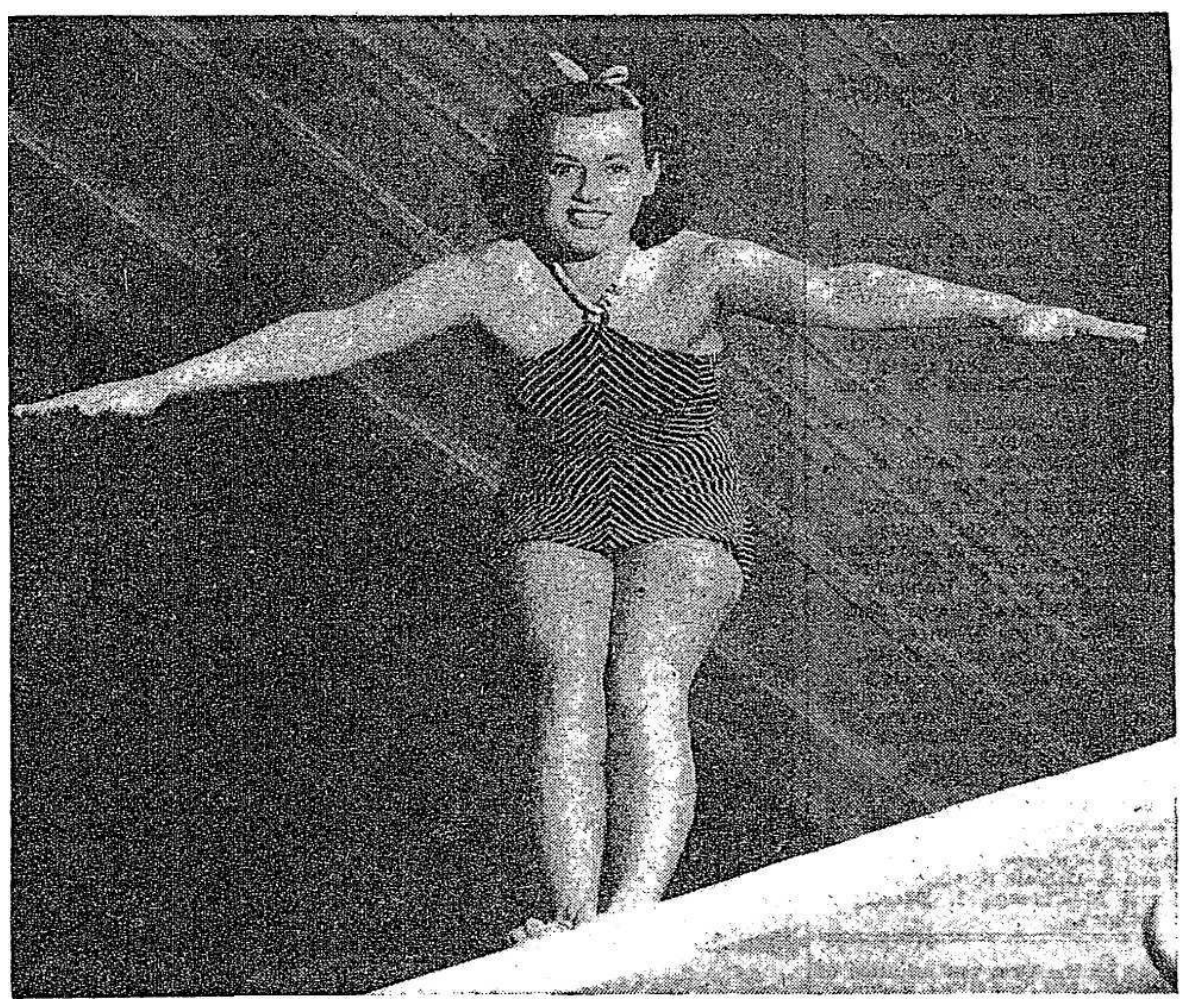

New York Times, 11 avril 1934. Voir infra la photographie du 25 juillet 1936 du Oldensburgh journal.

12 Pour toutes ces raisons, à partir de l'annonce officielle des champions sélectionnés pour Berlin faite à la mi-juillet 1936, Mrs Holm Jarrett devient l'une des athlètes américaines les plus en vue. Par ses exploits sportifs et sa touche «glamour » un brin sulfureuse, elle s'attire une horde de journalistes sur le pont du paquebot, le Manhattan :

Mrs. Eleanor Holm Jarrett, ready for her third Olympic back-stroke contest, had an aura of glamour all her own. She was literally covered with orchids from shoulder to waist, and the fact that she is the actual defender of a title brought autograph seekers, movie cameramen and amateur photographers in serried ranks about her ${ }^{16}$

Entre 1927 et 1936 on remarque donc qu'Eleanor est passée du bref entrefilet signalant son chrono record à de longs articles mêlant interview, récit sportif mais aussi biographie, la photographie venant bien vite mettre un visage sur ce nom. Gloire nationale, aussi bien des pages sportives que des pages "gossip ", et déjà aperçue dans quelques articles de la presse sportive étrangère, un événement durant la traversée va la rendre visible à l'échelle internationale.

\section{La croisière s'amuse}

14 En effet, pour reprendre la taxinomie de Nathalie Heinich, Eleanor Holm Jarrett va voir son capital de visibilité dû à ses propres capacités augmenter par le hasard, la propulsant alors grâce à un fait divers dans toutes les unes des journaux. Le 16 juillet la une du New York Times est consacrée au départ de la délégation olympique avec en page 23 une photo des athlètes sur le pont. Débute alors le feuilleton des JO, avec diverses chroniques concernant la traversée. L'une notamment, titrée, « Varied Programs to keep olympians diverted aboard ship ${ }^{17}$ » mentionne une certaine Mrs Sackett, « chaperonne de l'équipe 
des naïades ", dont le rôle est de les divertir à bord. Tous les athlètes présentant des talents artistiques sont réquisitionnés pour assurer le spectacle. Eleanor en qualité de chanteuse est bien sûr citée. Or cette petite chronique se charge quelques jours plus tard d'une ironie tragique quand les gros titres de la page sport annoncent le 24 juillet: «Eleanor Holm Jarrett dropped from Olympic Team for breaking training. " Comptant déjà parmi les personnages principaux de cette odyssée, c'est alors un véritable «spinoff » qui se met en place autour de la nageuse.

Alors que le Manhattan entre au port de Hambourg le samedi 23 juillet, le président du comité olympique américain, Mr Avery Brundage annonce une nouvelle qui suscite un raz de marée médiatique : Mrs Holm Jarret, que l'Amérique entière voyait déjà médaillée, est radiée de l'équipe, sommée de rendre son équipement et de reprendre un bateau en sens inverse. L'événement est d'abord raconté le 24 juillet par un envoyé spécial du journal, Arthur J. Dailey, qui assiste au discours de Brundage de la veille et tente de reconstituer la chronologie exacte de l'affaire. À cette heure dit-il, «on ne sait pas quelle règle a été enfreinte ${ }^{18}$ ", mais, en revanche, on apprend que Mrs Eleanor, malgré un premier avertissement, a eu une attitude contraire à l'éthique sportive, ce qui aurait forcé le comité à agir. L'article, en apparence neutre, désigne la jeune femme non pas sous les termes de «la nageuse » mais "la chanteuse de boîte de nuit et actrice de cinéma ", comme si le côté sombre de cet être ambivalent refaisait surface et expliquait son exclusion. La condamnation morale se lit également en filigrane dans d'autres journaux, par exemple dans le Oldensburgh journal qui titre une photo sur laquelle la nageuse ressemble à une danseuse de cabaret : "Now she is sorry ${ }^{19}$ ", sous-entendant à peine un «c'est un peu trop tard... »

Pendant près d'un mois, tous les jours plusieurs articles du New York Times - et l'ensemble de la presse quotidienne américaine - reviennent sur « l'affaire » qualifiée de «cause célèbre ${ }^{20}$ ». Les lecteurs américains suivent ainsi les différents épisodes du «feuilleton» de l'été: tout commence par une analepse de la traversée maudite. Que s'est-il réellement passé à bord ? Cette exclusion est-elle justifiée ? Plusieurs témoignages entrent en contradiction: une version glamour décrit l'affaire avec légèreté. La jeune femme, habituée au monde de la nuit, fait fi du couvre-feu officiel pour participer à une soirée en première classe et boire du champagne en compagnie de journalistes et d'artistes, comme le dramaturge Mac Arthur. Malheureusement elle est surprise par la chaperonne Mrs Sackett qui la dénonce auprès du comité. Au contraire le témoignage du médecin de bord, parlant de "poivrot» («boozer»), de coma éthylique et même de dépendance alcoolique, donne un tout autre éclairage et revient sur le rôle exemplaire que les athlètes sont censés tenir. En agissant ainsi, c'est toute la réputation de l'équipe américaine qui est entachée, d'où la nécessité de sévir. A force de versions multiples, s'élabore le "pilote", épisode inaugural de la série, qui se cristallise autour d'un symbole : le champagne.

17 Cependant, une autre question permet de maintenir le suspense : la décision d'Avery Brundage sera-t-elle définitive ? Changera-t-il d'avis durant les quelques jours qui restent avant l'ouverture des jeux ? Plusieurs articles notent par exemple que le nom de l'athlète n'a pas été rayé de la liste... et que cent cinquante sportifs ont signé une pétition de soutien en descendant du bateau! Sous le titre "Mrs Jarrett's plea denied, but she's in Berlin hopeful another chance ${ }^{21}$ ", on suit avec attention les efforts de Mrs Jarrett afin d'être réintégrée à l'équipe : on raconte ses larmes, on évoque la possibilité d'un pardon... peine perdue. Le comité olympique reste inflexible et ordonne le rapatriement de la 
nageuse. Sur ces entrefaites, entre en scène un nouveau personnage, le mari qui, resté aux Etats-Unis, décide de venir soutenir sa femme sur le sol allemand, crie à l'injustice et menace le comité de poursuites judiciaires ${ }^{22}$. Y aura-t-il procès ? Nouveau rebondissement dans l'intrigue : Eleanor passe à l'offensive et contre-attaque. Le New York Times publie une interview et un texte signé de sa main dans lesquels elle accuse non seulement les membres du comité de l'avoir transformée en bouc émissaire, mais encore de s'être euxmêmes adonnés à des pratiques peu recommandables à bord !

\section{She Claims Committee Members 'Disgraced' Themselves on Boat. \\ TELLS OF 'SHOCKING' SHOW' \\ Calls Kirby's Handling of \\ New York Times, 26 juillet 1936.}

18 L'auxiliaire modal du titre "she will see the Olympics ${ }^{23}$ " semble insister sur le pied de nez fait à la décision de Brundage : elle restera bien à Berlin et assistera aux JO, non pas comme athlète, mais comme journaliste ${ }^{24}$ ! En effet, une agence du groupe Hearst lui propose de couvrir l'événement, moins pour ses talents de plume que pour le tapage médiatique que cette nouvelle entraîne.

Tantôt repentante, tantôt rebelle, tantôt clémente, tantôt revancharde, Eleanor souffle le chaud et le froid dans ses déclarations et ménage régulièrement des retournements de situation qui la maintiennent dans les gros titres des journaux durant tous les JO et même au-delà. En effet, après la phase de sincères regrets, vient celle de l'affirmation d'un certain art de vivre, entrant pour le moins en contradiction avec l'esprit olympique ! Déjà, ce qui avait mis le feu aux poudres semble-t-il sur le bateau était le fait qu'elle s'était vantée de son entraînement " au champagne et au caviar ${ }^{25}$ » à ses petits camarades, ce qui ne manque pas de figurer en bonne place dans les titres du New York Times dès le 24 juillet. Le lendemain, le même journal publie une interview dans laquelle elle explique que la veille de la course pour la sélection olympique, elle avait fait la fête toute la nuit, ce qui ne l'a pourtant pas empêchée de remporter la course. Dans la même édition, son mari déclare de son côté que le comité « devrait donner plus de champagne à ses nageurs. Ainsi peut-être gagneraient-ils quelques courses». Le Syracuse Sport Journal publie même 
intégralement un article signé Eleanor Holm Jarret dans lequel elle prône la « relaxation » comme clé de la réussite! «Je bois une coupe de champagne et je fume une cigarette de temps en temps, je n'ai pas de mal à l'admettre, car au lieu de nuire à mon entraînement, cela n'a fait que l'améliorer "; et de donner quelques conseils à un autre nageur : « si Jack s'était accordé quelques verres de bière ou de champagne, ou quelques soirées dansantes, il serait maintenant au meilleur de sa forme!»

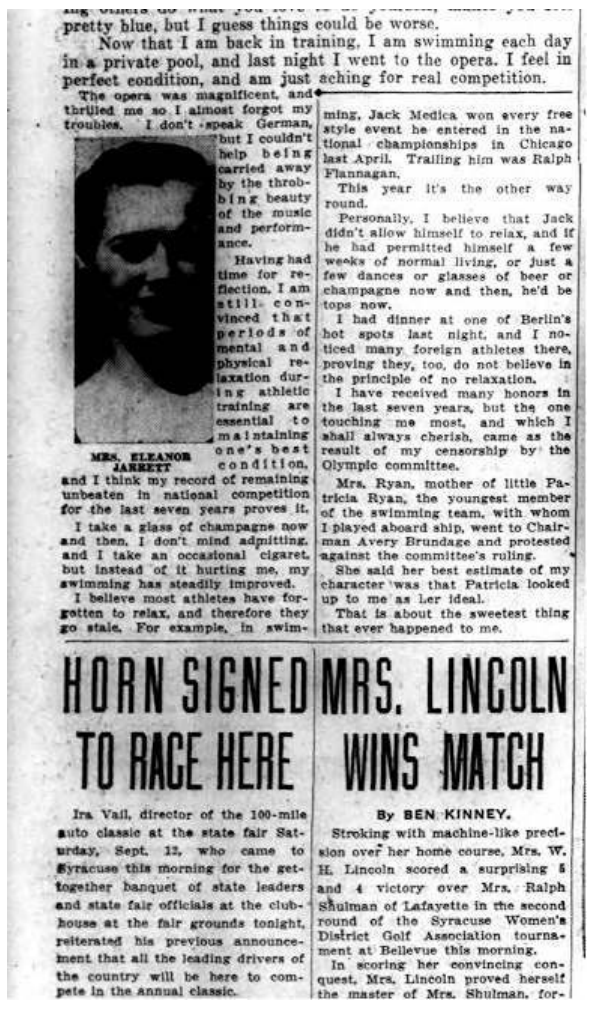

Syracuse Sport Journal, 29 juillet 1936.

Ces déclarations retiennent particulièrement l'attention de la presse française qui très certainement s'en inspire mais retranscrit de façons variées les propos de la nageuse. Le Figaro du 27 juillet donne ainsi en une un demi-aveu en lui faisant dire : « Evidemment, j'étais un peu gaie, mais je ne pensais pas que cela pût nuire à mon entraînement ${ }^{26}$ »; dans Le Petit Parisien, elle répond franchement à Brundage que « le champagne fait partie de son training ${ }^{27} »$, le journaliste reprenant un terme anglais pour faire couleur locale ; enfin quinze jours plus tard, L'Auto propose un " sensationnel reportage dans les coulisses de la délégation américaine ${ }^{28} »$, dans lequel la traduction semble prendre quelques libertés pour expliquer le "secret » de la réussite de la «very exciting " Miss Eleanor Holm Jarrett : «J'aime le champagne, il ne me fait que du bien », «plus je bois, mieux je nage », et encore « si je ne buvais plus, je serais battue... » 


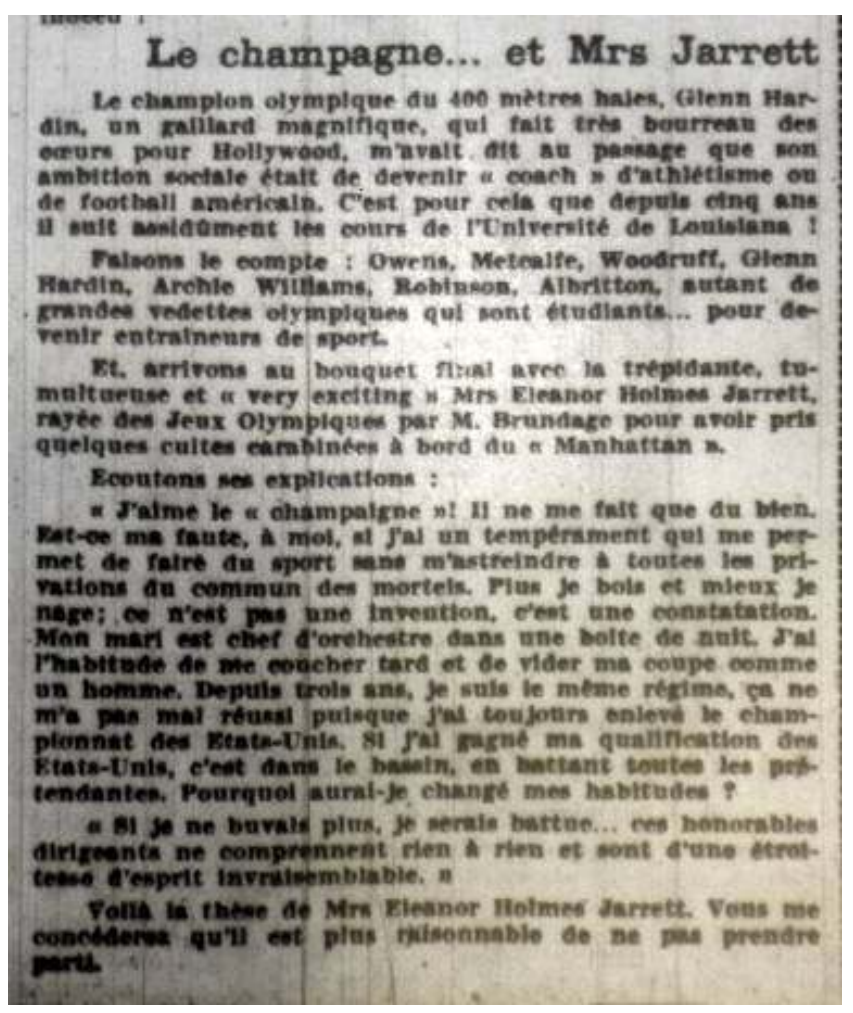

L'Auto, 10 août 1936.

21 En outre l'histoire tourne rapidement en affrontement entre deux personnages, Brundage versus la nageuse, devenant deux figures antinomiques, l'Ordre contre la Liberté, et une récupération politique discrète semble alors s'opérer. En effet, dès le 26 juillet, le New York Times publie une lettre de soutien à la nageuse signée du comité "Move the Olympics » qui s'est battu pour boycotter les Jeux face à Brundage dont les idées sont clairement pro-germaniques. Sans le vouloir, Eleanor devient ainsi un symbole d'insoumission et d'indépendance, face à l'autoritarisme, et la manière de rendre compte de l'incident du bateau devient une façon de prendre position sur l'échiquier politique. Ainsi selon le journal, l'affaire fait la une de la presse allemande. Celle-ci soutient unanimement la décision de Brundage, se déclare choquée du «laisser-aller de la délégation américaine » et en profite pour donner une belle leçon de morale au monde entier sur l'incompatibilité absolue du monde du spectacle avec la rigueur et la discipline qui règnent dans le sport ${ }^{29}$. 


\section{REICH PRESS BACKS \\ U. S. BODY'S ACTIION}

Dropping of Mrs. Jarrett is

Major Sensation and Front-

Page News in Berlin.

\section{EXPERTS GET A SURPRISE}

\section{Are Astounded by Americans' \\ Happy-Go-Lucky Attitude \\ Toward the Games.}

On vous a entretenu de la vie un peu trop joyeuse menée sur le Manhattan le « manhattan » est un excellent cocktail - par la délégation américaine aux Jeux olympiques. On buvait, paraît-il, trop de champagne. Cela a valı à la championne olympique, Mlle Eleanor Holm, d'être radiée, a son arrivée à Berlin, de la représentation des U.S.A. aux J.O. C'est peutêtre sévère, pour un hommage un peu trop sensible rendu à notre vin. Mais, ce qui est extraordinaire, c'est que Mlle Eleanor Holn était la seule Anéricaine qui eût une chance devant les Australiennes et les japonajses. Celles-ci ont dû admirer particulièrement ce hara-kiri américain.

Et voilà donc le vertueux esprit olympique qui souffle maintenant de l'Oues! ! Il nous vient d'Amérique. On aura tout va! Et les C.O. européens en petuent prendre de la graine.

\section{Jeän de LASCOUMETTES.}

New York Times, 25 juillet 1936 ; Match, 28 juillet 1936.

$\mathrm{Au}$ contraire la presse française semble plutôt prendre parti pour la jeune femme, en narrant ses exploits éthyliques sur un ton goguenard ou ironique. C'est l'esprit français de la gaudriole mais aussi celui de la fronde que semble incarner la jeune femme. "C'est peut-être un peu sévère, pour un hommage aussi sensible à notre vin " s'exclame Jean de Lascoumettes dans L'Intransigeant. Le Figaro témoigne aussi de son soutien dans son titre 
«la triste aventure d'une championne américaine de nage ${ }^{30}$ ", évoquant « deux ou trois malheureuses coupes de champagne ", version édulcorée du drame, ou encore émettant un jugement de valeur sur la décision du comité olympique: pour eux elle a été " malheureusement» radiée. Enfin, la presse américaine affiche clairement sa désapprobation vis à vis de Brundage en recourant à une anecdote historique célèbre qui compare Eleanor à un héros national :

When some one charged Gen. Grant, in the President's hearing, with drinking too much liquor, Mr. Lincoln, recalling Gen. Grant's successes, said that if he could find out what brand of whisky Grant drank, he would send a barrel of it to all the other commanders. ${ }^{31}$ "star », comme sur ce cliché du New York Times, « Mrs Jarrett sunning herself », où on la voit prenant le soleil sur le Manhattan, alors que sur la composition, les autres athlètes sont montrés en plein entraînement !
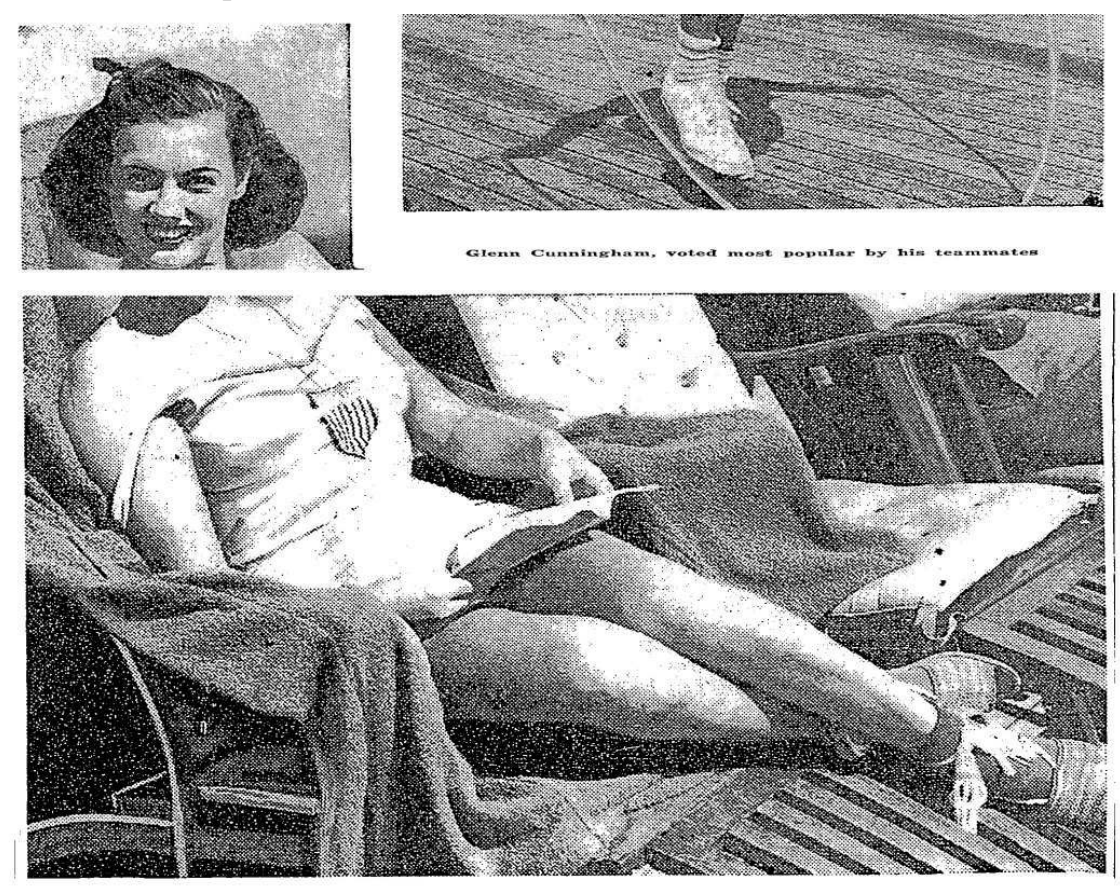

Mrs. Eleanor Holm Jarrett sunning herself. This was before she was ousted from the team because she likes champagne.

New York Times, 28 juillet 1936.

La presse américaine profite de sa présence sur place pour recueillir ses impressions sur les Jeux, comme dans l'affaire des sifflets dans le public visant les Américains lors de la cérémonie d'ouverture. Ayant assisté à l'incident, elle n'hésite pas à donner son avis, « 
Mrs Jarrett gives views ${ }^{33}$ ", étant persuadée que l'orchestre allemand s'est mis à jouer pour couvrir les huées du public et ainsi éviter l'incident diplomatique. Mais sa présence dans la tribune de la presse est aussi l'occasion de plusieurs récits aux résonnances tragiques, par exemple l'article sous-titré « Press Gallery Tragedy », dans lequel on la voit s'éloigner pour pleurer à chaudes larmes, alors que la délégation américaine s'apprête à passer sous ses yeux ${ }^{34}$.

Connue grâce à la presse, elle est aussi reconnue à Berlin, les journaux américains mettant en scène la joie qu'éprouvent les gens à la voir « pour de vrai». Ainsi, une chronique du 29 juillet relate la rencontre inattendue de la jeune femme avec le prince couronné Guillaume de Prusse, «l'homme qui aurait dû diriger l'Allemagne ${ }^{35}$ ». Se produit ici une inversion comique puisque c'est le prince qui reconnaît "la célèbre nageuse ", et non l'inverse. S'ensuit la visite guidée du Palais royal par son altesse. Cette scène est fortement fictionnalisée, notamment par le discours direct qui permet d'accentuer l'écart social entre le prince et la nageuse, dont le parler se veut populaire, par exemple avec l'emploi systématique en début de phrase de l'interjection «Gee!»: «Eh bien! Maintenant je peux bien oublier que j'ai été exclue de l'équipe ! C'est la première fois que je rencontre un membre d'une famille royale. Jamais je n'aurais pensé qu'un prince pouvait agir avec autant de naturel $!^{36} »$. Riant de la naïveté et de la franchise d'Eleanor, les lecteurs américains pénètrent dans les coulisses de l'histoire grâce à ce petit personnage populaire. Or le même jour dans le même journal, une brève raconte aussi comment la jeune femme, ayant voulu aller nager, est reconnue au bord du bassin par des Allemandes euphoriques, à qui elle donne ensuite une leçon de natation. Enfin la presse française mentionne très souvent la présence de Mrs Jarrett : on admire ses « longs cils » à la tribune des journalistes ${ }^{37}$, on la croise à une réception donnée par Goering, on décrit ses tenues affriolantes. Cette visibilité mise en abyme dans la presse donne l'impression d'une omniprésence de la jeune femme.

Célèbre d'abord par son mérite personnel, Eleanor est victime d'un drame qui paradoxalement redouble sa visibilité et réoriente sa carrière publique. Jouissant au moment des Jo de Berlin d'un nom et d'une image, elle ne cherche pas à enrayer le scandale mais au contraire va l'exploiter au maximum, comptant sur «les moyens techniques de mise en visibilité pour fabriquer et entretenir son capital ${ }^{38} »$.

\section{Un dernier pour la route}

La fin des Jeux olympiques ne sonne pas pour autant la fin d'Eleanor story. En effet, malgré son absence des podiums, la jeune femme fait un retour triomphal au même titre que les athlètes médaillés : deux photos, une lors de son départ à Hambourg en compagnie de son mari, l'autre à l'arrivée à New York avec sa mère, illustrent bien le fonctionnement de la machine médiatique. Alors que ces images ne sont porteuses d'aucune information nouvelle, elles figurent cependant en bonne place, la visibilité de la jeune femme pouvant être qualifiée cette fois d'endogène : elle est connue par sa notoriété, c'est parce qu'elle est médiatisée qu'elle continue à l'être. Cette vie médiatique va connaître plusieurs états : d'abord intensive jusqu'en 1939, puis de plus en plus espacée, mais persistante, jusqu'en 2004, date de son décès; enfin posthume, toujours grâce à la presse qui convoque ponctuellement ce "personnage ", mais aussi grâce à la littérature qui s'en empare en 2012. 


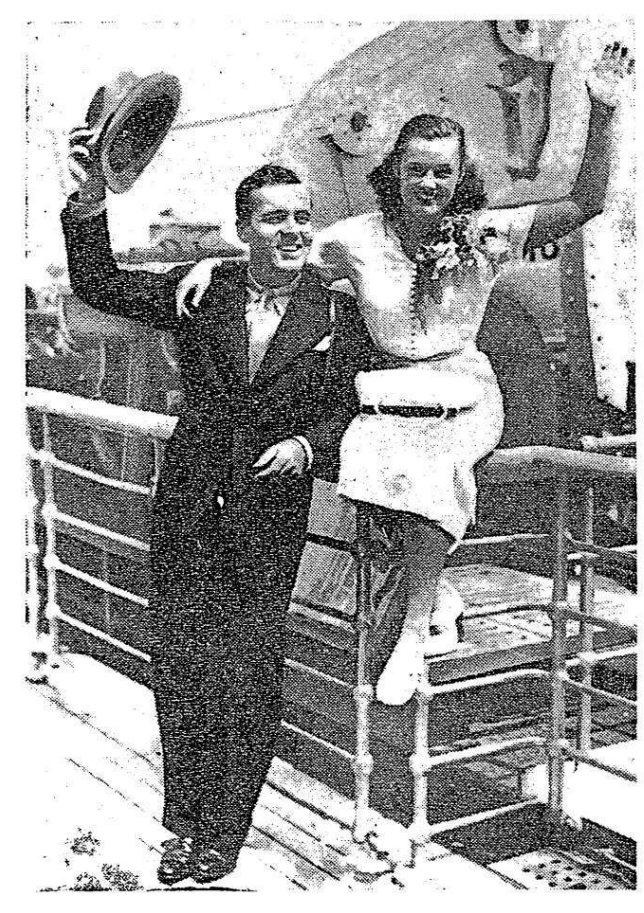

Mrs. Eleanor Holm Jarrett, swimming star who was world Photo.
the American team, with her husband aboard the Bremen.

Ogdenburgh Journal, 22 août 1936, p. 1.

29 À son retour sur le sol américain et pendant environ trois années, Eleanor colonise la presse par de multiples biais et dans des espaces divers : sports, social news, amusements... et semblant jouer la carte de la quantité plus que de la qualité. Le premier est la continuation de l'histoire des Jeux olympiques. On réchauffe, on fait durer, on reformule, sans qu'il y ait vraiment quelque chose de neuf à raconter. Deux éléments sont récurrents : la rivalité avec Brundage et ... le champagne! Ainsi le 21 août, le New York Times titre-t-il « Mrs Jarrett, back, does not plan any legal action - still likes champagne » tandis que le lendemain, le Ogdensburg Journal inscrit en une, juste en dessous des nouvelles internationales de la guerre d'Espagne : « Eleanor Jarrett Returns Bitter Against Brundage » 


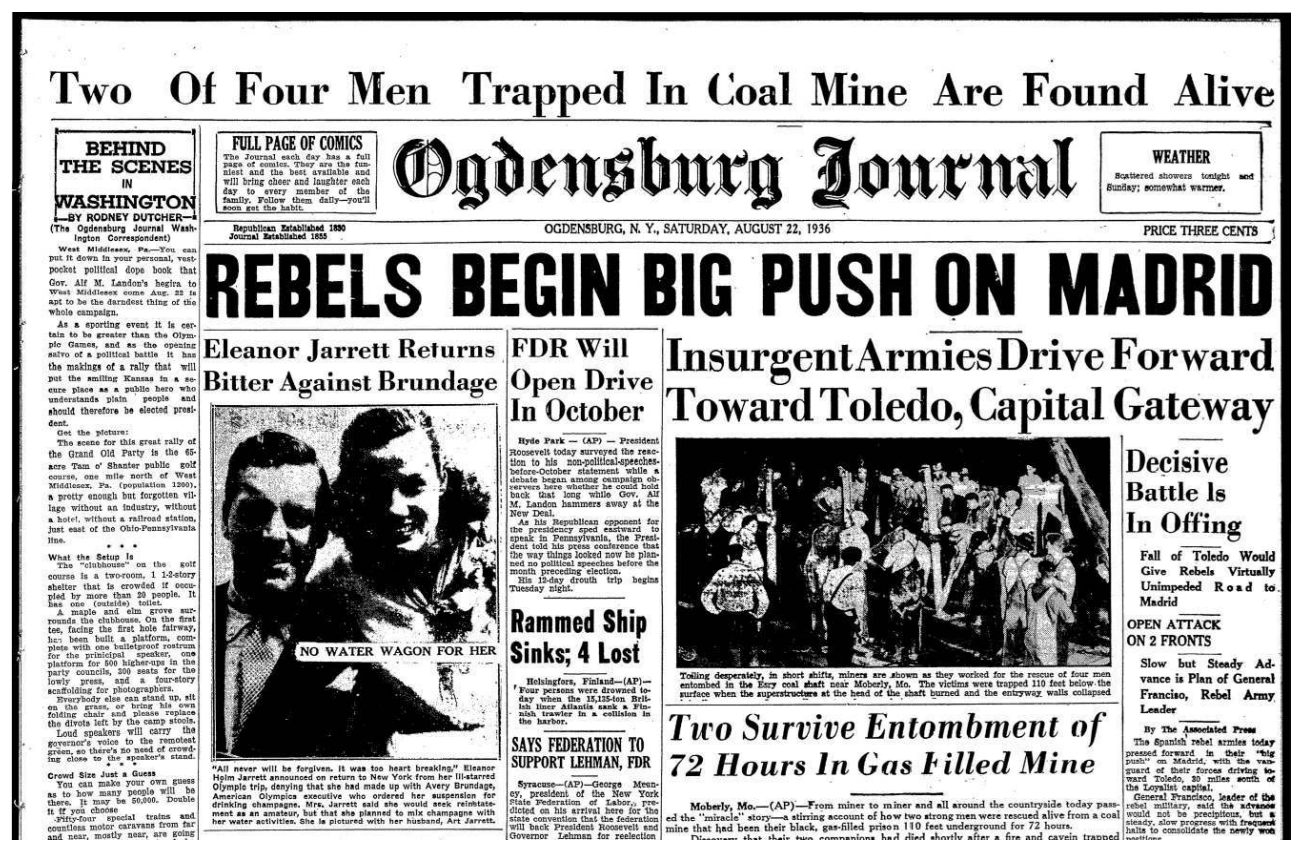

Ogdensburg Journal, 22 août 1936.

30 L'information est contradictoire et semble chercher à relancer la polémique. Quant au commentaire "No water wagon for her", il fait écho de manière humoristique au penchant de la nageuse pour les boissons alcoolisées.

31 Peu à peu, le contexte d'origine semble passer au second plan, Eleanor n'étant plus que "The Champagne girl ${ }^{39}$ ». Alors qu'Eleanor participe à la cérémonie d'inauguration d'un avion, The Advanced News détourne cette histoire pour présenter l'information : « Oh ! Mr Brundage ! Look it ! that's real champagne Eleanor Jarrett is wasting in the christening of the flying box car TWA's first all freight air service plane from NY to Chicago ! ${ }^{40}$ » Enfin dans la revue sportive de l'année 1936, pour laquelle le journaliste imagine le repas de Noël des athlètes célèbres, c'est ainsi « a succulent dish of caviar with champagne on the side $^{41} »$ qui est servi à la nageuse. 


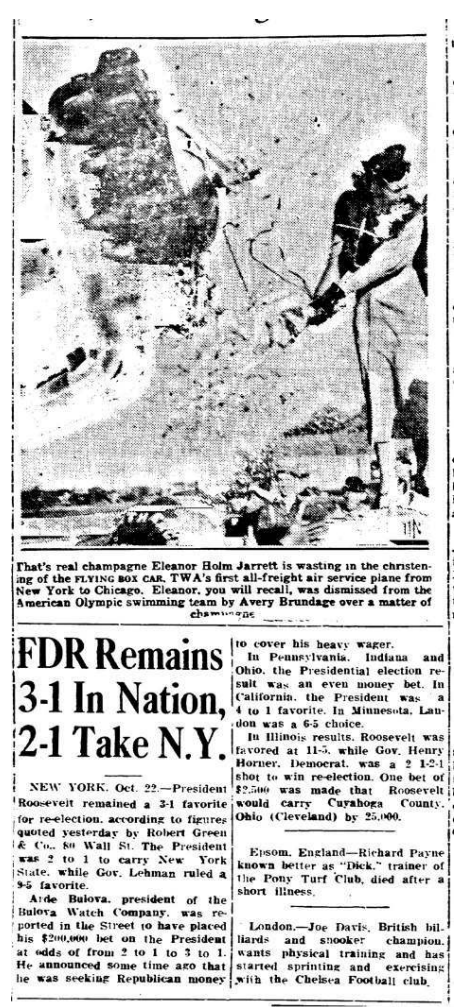

The Advanced News, 23 octobre 1936.

La presse suit également Eleanor de près dans toutes ses nouvelles activités. Même si la sportive ne nagera plus jamais en compétition officielle, renonçant définitivement, après quelques batailles, à son statut d'amateur, la notoriété acquise lors des Jo lui ouvre les portes de nombreux projets hétéroclites mais tous fortement lucratifs. Ainsi dès septembre 1936 un festival de natation est organisé en son honneur par un journal, le New York Evening Journal, et porte son nom. Sa carrière de comédienne explose également durant cette période, avec plusieurs tournées qu'elle enchaîne avec son mari. Dans un article intitulé « Mrs Jarrett prospers : doing well on stage since her return from Europe ${ }^{42}$ ", elle se vante de gagner beaucoup d'argent. Un important projet cinématographique lui est aussi proposé à cette période, Tarzan's revenge, qui lui assure alors une place dans les rubriques "Amusements » et "Theatre " dans les journaux, ainsi que quelques brèves dans la presse française. Mais c'est certainement sa participation au spectacle Aquacade, vu par des milliers d'Américains, qui lui vaut le plus d'interviews et de couvertures dans les magazines. Enfin elle assure aussi personnellement sa présence dans les médias en collaborant sporadiquement à des projets de presse, comme par exemple une série illustrée dans laquelle elle écrit une méthode pour apprendre à nager le crawl ! Diffusée à la une du Plattsburgh Daily Republican, on voit des clichés montrant les divers mouvements des bras et des jambes, assortis de conseils de l'ancienne championne olympique. Ainsi Eleanor, tel un pantin, est exploitée de façon intensive par les médias qui la transforment en produit de consommation, bien loin de sa première image simple de petite nageuse. Exposée ainsi dans ses moindres faits et gestes, elle est également dépossédée de sa vie privée. 


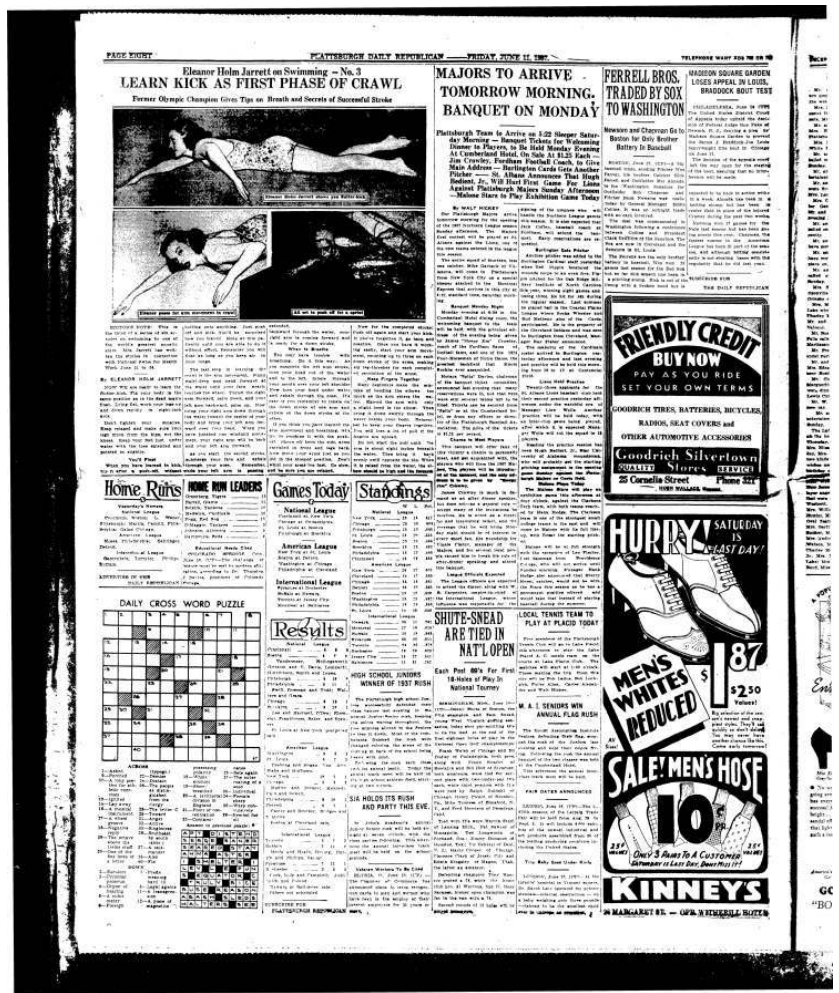

Plattsburgh Republican, 11 juin 1937, p. 1.

En effet Eleanor nourrit abondamment la chronique gossip, d'abord avec son mari Art, puis par leur rupture et sa liaison avec le producteur de l'Aquacade Billy Rose. La presse de l'époque permet de reconstruire le drame amoureux presque heure par heure! Le 21 juillet 1937, le New York Times en page 4 annonce qu'elle va peut-être se séparer du chanteur, tandis que dans le Plattsburgh daily press, l'article « it may be love, but it isn't $\mathrm{Art}^{43}$ " annonce la rupture. Les photos du couple Eleanor/Art laissent place à celles d'Eleanor « et son Billy ${ }^{44} »$.

Plusieurs interviews sur la question permettent à la jeune femme de se plaindre du tapage médiatique autour d'elle et de Billy Rose. Elle en a assez de voir son nom dans les gros titres accolé à celui de son producteur, qui lui-même déclare ne rien savoir sur le sujet. «Je suis juste le producteur du spectacle dans lequel elle joue, je suis marié à Miss Brice [une actrice] et ai bien l'intention de le rester. » Protestations qui, au passage, fournissent matière à article.... Alors que sa carrière en tant qu'actrice n'est que peu relayée à l'étranger, ce scandale récolte plus d'échos, comme ici dans la rubrique « histoires comme ça » du Petit Parisien : 


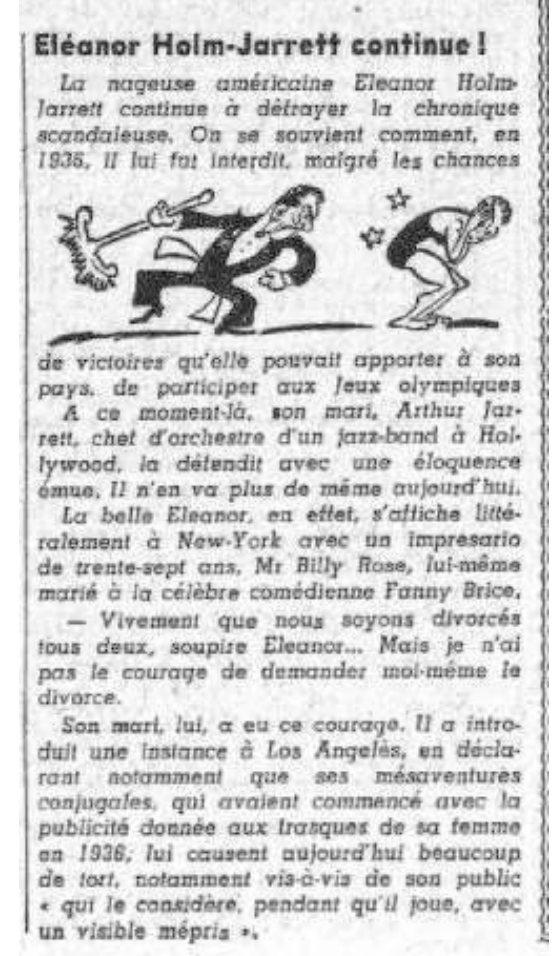

Le Petit Parisien, 8 décembre 1937, p. 6.

Enfin dès 1937, la presse propose un retour réflexif sur la trajectoire médiatique de la jeune femme, dans un principe de médiatisation endogène. Le 2 août 1937 le Citizen Advertiser publie une frise intitulée "Splash », onomatopée renvoyant à la fois au bruit du plongeon et au fait de faire sensation. L'article propose un résumé en image de sa carrière médiatique, tout comme "A day with Eleanor Jarrett ", sorte de roman photo où les six clichés sont censés recomposer de manière chronologique sa vie: jeune fille, elle s'entraîne pour devenir championne, avant de devenir une star convoitée. 


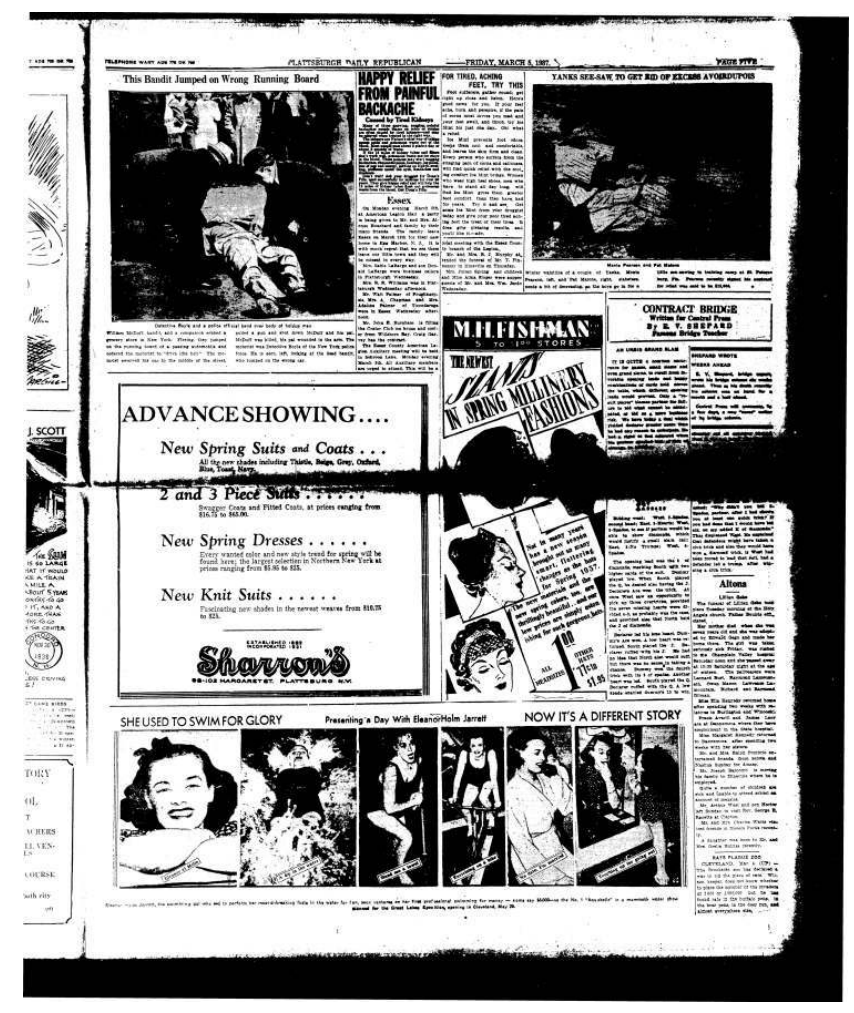

PLATTSBURgH DAILY REPUBLICAN, 5 MARS 1937

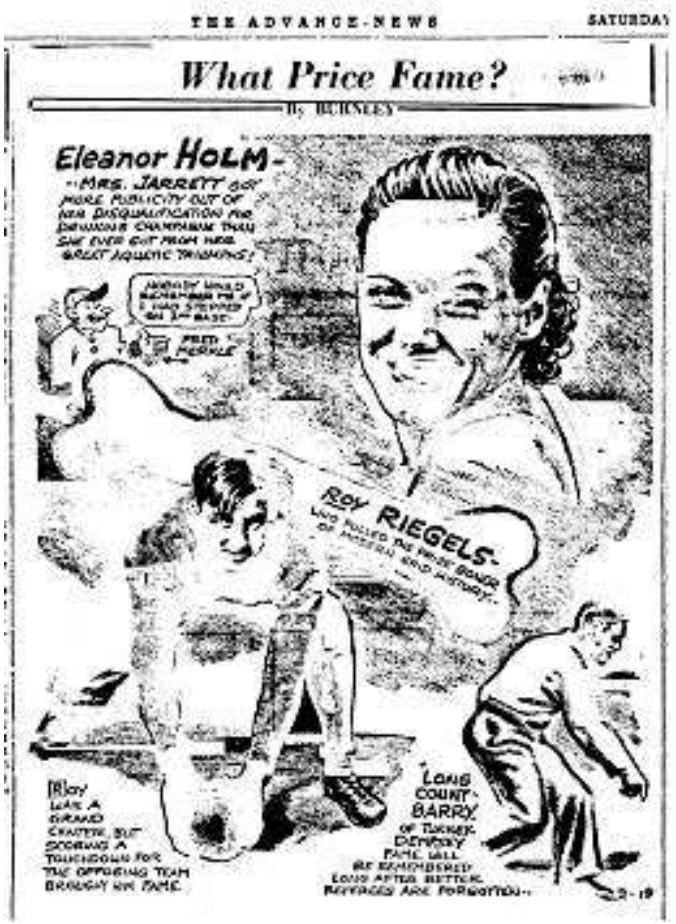

The Advanced News, 19 septembre 1936.

«La renommée, à quel prix ? » c'est aussi le titre d'un article de The Advance news où son illustration figure en bonne place, avec comme commentaire: "C'est bien sa disqualification qui causa à Mrs Jarrett le plus de succès, malgré tous ses triomphes en natation. » 
Veritable personnage médiatique, cette omniprésence dans la presse américaine s'estompe après 1939. Pourtant Eleanor ne disparait pas complètement. Faisant partie de la légende olympique nationale, elle est régulièrement conviée. Aussi la presse moderne s'emploie-t-elle à reconnaître dans la " grande dame des Jeux olympiques ${ }^{45}$ » la pétillante et rocambolesque jeune femme d'antan. Les questions, cinquante ans plus tard, n'ont pas changé, et tournent encore autour de Brundage et du champagne. Un article du New York Times de janvier 1994 titre ainsi : «The champagne's girl who says no to champagne »; et lors de la diffusion d'un film documentaire sur les femmes dans le sport ${ }^{46}$ à la Maison Blanche en 1999, la rencontre d'Eleanor avec le président Bill Clinton semble rejouer la scène du Prince Guillaume, avec la même liberté de propos. Comme en 1936 à la tête couronnée, elle déclare au président qu'il a un physique avantageux ("you're a good looking dude ») et qu'il devrait songer à faire du cinéma! Elle-même semble être très lucide sur sa carrière médiatique, tout en continuant à la mener de façon habile. Ainsi elle avoue qu'elle doit sa renommée à Brundage: «oui, je suppose que si je suis toujours connue, c'est à cause de tout ce clinquant et de Brundage ${ }^{47}$ ». Mais elle n'en oublie pas moins de le traiter de « vieillard répugnant » (« dirty old $\left.\operatorname{man}^{48} »\right)$, réactivant l'ancienne querelle, et n'hésitant pas à servir un ragot croustillant : « une des théories est qu'il en pinçait pour moi, mais que je l'ignorais ${ }^{49}$ "! De même évoque-t-elle sans complexe son entretien privé avec Hitler, ou la broche offerte par Goering, participant ainsi elle-même à la perpétuation de la légende. La vénérable Eleanor, expliquant qu'on la «ressort d'un tiroir tous les quatre ans ", pour suivre le calendrier olympique, n'en participe donc pas moins au cirque médiatique, qu'il s'agisse simplement de raconter son histoire à l'occasion d'un documentaire sur les Jeux olympiques, ou de la faire résonner avec une affaire d'actualité, comme dans le cas de la disqualification pour dopage de la sportive Tonya Harding ${ }^{50}$, en 1994 dans laquelle elle est amenée à témoigner comme ancienne athlète ayant été exclue des jeux. Enfin à sa mort en 2004, nombreux sont les journaux, généralistes comme spécialisés, à raconter une énième fois ses péripéties. Le New York Times termine son article hommage par l'une des dernières interviews d'Eleanor, sur ces mots emblématiques : «And I don't drink Champagne anymore. Just a little dry white wine.»

Les médias ont fait d'Eleanor un "personnage » : d'abord enfant prodige, puis hérö̈ne d'un drame, enfin vedette libérée, et même vieille dame bavarde et sympathique. Ce n'est donc presque pas étonnant de la retrouver comme héroïne d'une fiction sous la plume de David John, un auteur britannique, en $2012^{51}$; ce qui l'est plus en revanche, c'est le genre dans lequel s'inscrit Flight from Berlin ${ }^{52}$. Il s'agit en effet d'un roman policier dont l'intrigue est fortement liée au contexte politique des JO de Berlin, amenant une dernière reconfiguration à la persona d'Eleanor, en la transformant en héroïne engagée dans la lutte contre le nazisme, ce que semble illustrer la couverture.

Le roman débute par l'annonce de sa sélection dans l'équipe nationale de natation, ce qui réjouit la jeune fille, qui ne pense qu'à nager et à s'amuser en compagnie de son mari chanteur. Seule ombre au tableau, son père, un sénateur faisant partie du camp politique pour le boycott des jeux, qui la sermonne avant son départ pour son manque d'implication idéologique et pour ses choix de vie. Le récit de la traversée la montre rebelle, développant ainsi toutes les bribes médiatiques vues précédemment, et notamment la fameuse scène du champagne : invitée sur le pont supérieur par Charlie Mac Arthur et sa brillante petite compagnie, elle se distingue en chantant Let's misbehave de Cole Porter et ne se démonte pas lorsqu'apparaît sa chaperonne Mrs Hacker -

Belphégor, 15-1 | 2017 
remarquons la ressemblance avec le vrai nom de Miss Sackett -, qu'elle ridiculise en faisant montre de beaucoup d'esprit : «Friends, do we need to go to Germany to see a notorious dictator with a moustache? We have our very own right here. Folks, meet our team chaperone, Mrs Eunice Hacker ${ }^{53}$.»

Mais, à cause d'un chagrin d'amour, elle cherche refuge dans les coupes de champagne que lui tend William Hearst Junior jusqu'à sombrer dans un profond brouillard. David John, on le voit, en fait donc un personnage audacieux, impertinent et même engagé. En effet, choquée de certains propos pro-germaniques sur la « race future » dans le discours officiel de Brundage sur le bateau, elle manifeste ouvertement son désaccord et commence à comprendre le point de vue de son père. L'épisode du champagne est donc montré comme un prétexte permettant au président du comité olympique d'éliminer la fille de son rival, qui va alors entrer en lutte contre le parti nazi, en devenant journaliste à Berlin et en menant une enquête sur la corruption des jeux en compagnie d'un intrépide reporter - anglais, cela va de soi. Ainsi son exclusion de l'équipe nationale devient presque un acte de résistance : l'épisode du champagne est minimisé, excusé, et sonne les débuts de l'engagement d'Eleanor dans la lutte contre Brundage, qui s'est laissé corrompre par ambition.

David John propose donc une libre réinterprétation des scandales des JO de Berlin du côté de l'équipe américaine en reliant notamment l'exclusion injustifiée des deux coureurs juifs dans la course de relais à celle de la nageuse. Eleanor surprend en effet dans l'intrigue une conversation secrète entre Brundage et un homme au fort accent allemand qui promet au dirigeant la direction du comité international olympique à la condition qu'il se débarrasse de ses deux champions juifs. L'auteur du roman policier rend donc explicites les rumeurs que les médias de l'époque avaient exprimées plus ou moins haut et transforme concrètement Eleanor en chantre de la liberté et des droits de l'homme.

Impossible de revivre les jeux de Berlin à travers la presse française sans croiser à la tribune ou lors d'un banquet, au stade ou dans la ville, ce petit personnage d'Eleanor Holm Jarrett, qui avec son anecdote, ses longs cils et ses robes affriolantes apporte une touche légère et colorée dans ce climat souvent tendu et peu propice au rire. Cependant, malgré une apparition fulgurante sur la scène internationale à cette occasion, son histoire s'écrit davantage à l'échelle nationale: nageuse aux capacités exceptionnelles, dotée d'une beauté irrésistible et d'un caractère affirmé, elle voit s'emballer la machine médiatique à la suite de l'épisode de juillet 1936, qui la propulse au rang de star, rang qu'elle va s'employer à conserver le plus longtemps possible. D'abord petit poisson des bassins, puis sirène des night-clubs, son image se diffracte à l'infini pendant une courte période et se brouille (chanteuse, comédienne, actrice, journaliste, femme adultère, naïade de l'Aquacade, icône publicitaire), avant d'être figée après une période de relatif oubli par les médias contemporains et la littérature, qui ne conservent que quelques traits caractéristiques dignes de figurer dans la légende, dont l'impertinence et...le champagne. 


\section{NOTES}

1. Nathalie Heinich, De la visibilité, Excellence et singularité en régime médiatique, Gallimard, 2012, 593

p.

2. Nous travaillons essentiellement sur le New York Times et quelques journaux en ligne sur le site de la Fulton History Society.

3. New York Times, 21 mai 1927.

4. Id.

5. New York Times, 28 juin 1928.

6. Match, 24 avril 1928.

7. Il est amusant de voir que Henry Musnik propose le 7 mars 1933 un nouveau « récit des origines " dans lequel cette fois la fictionnalisation est totale : le journaliste en effet raconte comme s'il y avait assisté le moment où Eleanor décide de devenir championne olympique. Alors qu'elle rencontre dans son club de natation l'équipe américaine de retour des Jeux, elle alpague ses aînées, leur demandant la recette du succès, et déclare qu'elle fera pareil. Ainsi la championne est déjà personnage de fiction, représentée comme une petite fille au caractère bien trempé et à la langue bien pendue, maîtresse de son destin.

8. Match, 24 avril 1928.

9. Nathalie Heinich, op. cit., p. 203.

10. Id.

11. Match, 7 mars 1933.

12. The Advanced News, 28 septembre 1935.

13. New York Times, 10 mai 1936.

14. Maribel Y. Vinson, « News of Women In Sports », New York Times, 10 Mai 1936.

15. Id.

16. Maribel Y. Vinson, « News of Women in Sports ", New York Times, 19 juillet 1936.

17. New York Times, 11 juillet 1936.

18. New York Times, 25 juillet 1936.

19. Ogdensburgh Journal, 24 juillet 1936.

20. On retrouve l'expression en français dans plusieurs journaux, dès le 14 septembre 1936 dans le New York Times.

21. New York Times, 25 juillet 1936.

22. Id.

23. New York Times, 27 juillet 1936.

24. New York Times, « Mrs Jarrett Joins Host of Writers at Olympics », 28 juillet 1936.

25. New York Times, 25 juillet 1936.

26. Le Figaro, 27 juillet 1936, p. 1.

27. Le Petit Parisien, 25 juillet 1936, « dernière heure », p. 3.

28. L'Auto, 10 août 1936.

29. New York Times, 25 juillet 1936.

30. Le Figaro, 27 juillet 1936, p. 1.

31. New York Times, 30 octobre 1936.

32. New York Times, 8 août 1936.

33. New York Times, 2 août 1936

34. New York Times, « Mrs Jarrett pay way to pool for the first time », 3 août 1936 
35. New York Times, 29 juillet 1936.

36. Id.

37. Voir dans ce numéro l'article collectif sur la presse française et Le Petit Parisien.

38. Nathalie Heinich, op. cit., p. 134.

39. New York Times, janvier 1994.

40. The Advanced News, 23 octobre 1936.

41. New York Times, « Sports of the Times », by Arthur J. Daley, 25 décembre 1936.

42. New York Times, 22 octobre 1936.

43. Plattsburgh daily press, 23 juillet 1937.

44. Ogdensburgh Advanced News, 17 novembre 1937.

45. Titre d'un article du New York Times, "Sports of the Times ", 3 juillet 1984.

46. Dare to Compete : the Struggle of Women in Sports, HBO, 1999.

47. New York Times, «Sports of the Times », 3 juillet 1984.

48. New York Times, janvier 1994.

49. New York Times, «Sports of the Times », 3 juillet 1984.

50. «The 'Champagne Girl' of '36 Can Empathize », 18 janvier 1994. La patineuse Tonya Harding a été exclue de l'équipe olympique pour avoir commandité l'agression de sa mère, qui par ailleurs la battait lorsqu'elle était enfant.

51. Ibid, «note d'auteur », p. $347:$ «It was the story of American Olympic swimmer Eleanor Holm and her antics on board the Manhattan in 1936 that was the catalyst to start writting. Holm had such spirit, beauty, and courage that in my mind she quickly inspired a fictional character in need of adventure "

52. David John, Flight from Berlin, Collins, 2012.

53. Ibid, p. 36.

\section{ABSTRACTS}

Qui est donc Miss Eleonor Holm Jarrett, athlète qui, le temps des JO de Berlin, devint la coqueluche des journalistes internationaux ? Cet article retrace la construction de cette curieuse figure médiatique qui, de la page sportive à la page «people» cumula différents « capitaux de visibilité », jusqu'à devenir un véritable personnage. En marge des grands événements sportifs, loin des enjeux politiques, et pourtant paradoxalement au cœur de la sociabilité de ces olympiades, l'étonnante trajectoire de la nageuse américaine exclue de la compétition pour une affaire de champagne offre un regard décalé sur l'événement au centre de ce dossier.

\section{INDEX}

Mots-clés: Visibilité, médias, fictionnalisation, Eleonor Holm Jarrett, jeux olympiques 1936 


\section{AUTHOR}

\section{AMÉLIE CHABRIER}

Amélie Chabrier est maître de conférences de littérature française à l'université de Nîmes. Elle a soutenu en 2013 à Montpellier III une thèse intitulée « les écrivains au prétoire. Chronique judiciaire et littérature au XIX siècle ». Elle a collaboré à la plate-forme Médias19.org sur la culture médiatique et le journalisme au XIX ${ }^{\mathrm{e}}$ siècle et a co-organisé en 2016 avec Marie-Eve Thérenty et la BILIPO le projet « Détective, fabrique de crimes? ", autour de l'hebdomadaire Gallimard de 1928, qui comprend une exposition itinérante (Paris, Montpellier, Nîmes), 3 journées d'étude et un livre (publication janvier 2017, éditions Joseph K.) 